\title{
CYP1B1-mediated Pathobiology of Primary Congenital Glaucoma
}

\author{
${ }^{1}$ Muneeb A Faiq, ${ }^{2}$ Rima Dada, ${ }^{3}$ Rizwana Qadri, ${ }^{4}$ Tanuj Dada
}

\begin{abstract}
CYP1B1 is a dioxin-inducible enzyme belonging to the cytochrome P450 superfamily. It has been observed to be important in a variety of developmental processes including in utero development of ocular structures. Owing to its role in the developmental biology of eye, its dysfunction can lead to ocular developmental defects. This has been found to be true and CYP1B1 mutations have been observed in a majority of primary congenital glaucoma (PCG) patients from all over the globe. Primary congenital glaucoma is an irreversibly blinding childhood disorder (onset at birth or early infancy) typified by anomalous development of trabecular meshwork (TM). How CYP1B1 causes PCG is not known; however, some basic investigations have been reported. Understanding the CYP1B1 mediated etiopathomechanism of PCG is very important to identify targets for therapy and preventive management. In this perspective, we will make an effort to reconstruct the pathomechanism of PCG in the light of already reported information about the disease and the CYP1B1 gene.
\end{abstract}

Keywords: CYP1B1 gene, Functional genomics, Glaucoma, Intraocular pressure, Pathobiology, Primary congenital glaucoma, Trabecular meshwork.

How to cite this article: Faiq MA, Dada R, Qadri R, Dada T. CYP1B1-mediated Pathobiology of Primary Congenital Glaucoma. J Curr Glaucoma Pract 2015;9(3):77-80.

Source of support: Nil

Conflict of interest: None

\section{CYP1B1 AND OCULAR DEVELOPMENT}

CYP1B1 is an important gene for developmental orchestra and plays ardent role in the embryonic development. It is a developmentally important gene having indispensible roles to play explicitly in ocular developmental biology. Important studies have reported the presence of CYP1B1 mRNA in a variety of fetal tissues ${ }^{1}$ and anatomical

\footnotetext{
${ }^{1,3}$ Student, ${ }^{2,4}$ Professor

${ }^{1,4} \operatorname{Dr}$ Rajendra Prasad Centre for Ophthalmic Sciences, All India Institute of Medical Sciences, New Delhi, India

${ }^{2}$ Laboratory for Molecular Reproduction and Genetics Department of Anatomy, All India Institute of Medical Sciences New Delhi, India

${ }^{3}$ Department of Laboratory Medicine, All India Institute of Medical Sciences, New Delhi, India

Corresponding Author: Tanuj Dada, Professor, Dr Rajendra Prasad Centre for Ophthalmic Sciences; All India Institute of Medical Sciences, New Delhi-110029, India, e-mail: tanujdada@ gmail.com
}

compartments-most importantly kidneys. This is to mention that these studies were silent about many details of the samples used to detect CYP1B1 mRNA in the fetal tissues. RT-PCR based studies on CYP1B1 expression have reported its constitutive expression in many fetal tissues, including brain, adrenal glands, liver and kidneys in the first and second trimesters. ${ }^{2}$ The expression of this gene almost ubiquitously in fetal tissues and anatomical compartments implies its importance in development and advocates its involvement in developmentally important disease which includes primary congenital glaucoma (PCG). Mutations in CYP1B1 gene or any other derangement precipitating its functional deficit, by this logic, may cause congenital malformations which include $\mathrm{PCG}^{3,4}$ and, by extension of this logic, other anterior segment disorders. What is important to note is that CYP1B1 null mice are, as such, normal ${ }^{5}$ but peculiarly display isolated defects in tissues relevant to PCG. ${ }^{6}$ Additionally, CYP1B1 has been found to be important in pregnancy maintenance because CYP1B1 polymorphisms are observed to be imperative in proper functioning of estrogen and progesterone receptors. ${ }^{7}$ A relevant point to note here is that $\mathrm{V} 432 \mathrm{~L}$ variant of the wild type has quadrupled $\mathrm{K}_{\mathrm{m}}$ for stereospecific 4-hydroxylation of $17 \beta$-estradiol which may be mediated through a drift in estradiol binding affinity. ${ }^{8}$

\section{PRIMARY CONGENITAL GLAUCOMA GENETICS AND CYP1B1}

Primary congenital glaucoma, a blinding disorder with onset at birth or during infancy, mainly presents with developmental abnormality in trabecular meshwork (TM) often coupled with other associated symptoms. Trabecular meshwork dysgenesis causes an obvious obstruction to the aqueous outflow precipitating increase in the IOP leading to ocular hypertension mediated optic nerve cupping and consequent loss of vision. ${ }^{9}$ Primary congenital glaucoma has autosomal recessive mode of transmission with varied prevalence $(1: 10,000$ in the western world to 1:1,250 among the Slovak Gypsies). ${ }^{10}$ Consanguinity has been found to be a fundamental mechanism for increased PCG prevalence in certain population like Saudi Arabians and Slovakian Gypsies. Primary congenital glaucoma is a complex disease which has been mapped to at least three genetic loci viz 
GLC3A (GLC abbreviates to glaucoma, three means the congenital form of glaucoma and the suffix A, B and C reveals the chronological order of the deciphering of these loci) chromosome $2(2 \mathrm{p} 21) ;{ }^{11}$ GLC3B-chromosome 1 (1p36); ${ }^{12}$ and GLC3C-chromosome 14 (14q24.3). ${ }^{13}$ The majority of PCG cases present with GLC3A involvement, specifically pointing toward CYP1B1 gene-based etiology.

\section{CYP1B1 AND PCG MECHANISM}

CYP1B1 is pivotal in the proper development of TM (the most important tissue with regards to PCG) ${ }^{14,15}$ and also in the in utero development of many other ocular tissues. ${ }^{16}$ The exact mechanism by which CYP1B1 leads to proper TM development is not known but its importance in development and maintenance of TM has well been opined. One important domain of our ongoing work is focused on this aspect of PCG pathogenesis. Some investigators hypothesize that it is either the metabolic conglomeration of some important unknown metabolite (indispensible for ocular development) or the elimination of some, yet unidentified, toxin (detrimental to proper eye development) that may be the mechanistic instrument of CYP1B1-mediated TM dysgenesis in PCG. However, a third mechanism can also be thought of in which CYP1B1 may be orchestrating the expression of some important genes relevant to anterior chamber formation. ${ }^{14,15}$ Mutations detrimental to the CYP1B1 function can, therefore, be thought of as critically pivotal in deranging the proper development of TM and subsequent reduction in aqueous outflow and consequent elevation of IOP. More than 140 mutations in CYP1B1 gene have been reported in PCG cases from all over the world till date $^{17,18}$ many of which have been exclusively reported to be present in PCG cases. ${ }^{4,14,19-27}$ Panicker et al recently reported eight mutations in CYP1B1 gene in Indian population many of which were present in the conserved regions and the functionally important regions (FIRs). ${ }^{28,29}$ We have also reported similar results from North Indian population. ${ }^{30,31}$ The complete mechanism of CYP1B1-mediated PCG pathogenesis has not been worked out but it has been proved beyond any reasonable doubt that CYP1B1 is one of the main genes involved in etiopathogenesis of PCG. Elucidation of the etiopathobiology of PCG is a severe need of the hour and can be done through functional characterization of the mutations that have been exclusively reported in PCG. Once the functional characterization of these mutations is accomplished, the vital molecular nodes in pertinent pathways can be identified and worked out which may lead to important interventions for effective treatments. For this reason, functional characterization of novel CYP1B1 mutations found in CYP1B1 cases is imperative and stands justified with a clinically bolstered rationale. ${ }^{32}$

\section{FUNCTIONAL INSIGHTS}

There are a number of ways to execute the functional characterization of these mutations but the most viable would be to express the wild and mutant types in bacterial expression systems, purify them and then compare their activities with respect to various metabolic processes including, but not limited to, estradiol metabolism, retinoid metabolism, arachidonate metabolism and melatonin metabolism (pathways crucially imperative in eye development). For such studies, heterologous expression of unmodified, full length human CYP1B1 is necessary. Our research group has already reported an easy and novel protocol for the heterologous expression of unmodified, full length human CYP1B1 in E. coli. ${ }^{33}$ Structural modeling should also be done to supplement such in vitro studies as they yield some important information that may prove pivotal in deciphering the mechanism of function loss in mutants in comparison to wild type of CYP1B1. One good news in this regard is that the crystal structure of CYP1B1 (507 amino acid stretch) has been recently reported. Its PDB ID is 3PMO [assessable at: http://www.rcsb.org/pdb/explore. do?structureId=3pm0]. Structure can be modeled using many softwares like MODELLER [assessable at: https:// salilab.org/modeller/download_installation.html]. The pathogenecity of each mutant can also be predicted by in silico methods like PolyPhen (Polymorphism Phenotyping) [assessable at: http://genetics.bwh.harvard. edu/pph2/] and SIFT (Sorting Intolerant from Tolerant) [assessable at: http://sift.jcvi.org/] analyzes. Not only this, stability of the mutant protein as compared to the wild type can also be checked with in silico methods. Many softwares are available for this purpose (e.g. SNAP2 and I-Mutant, etc.). Functional studies are important because the disease causing mutations are mostly present in the functionally important regions (FIRs). We have recently reviewed the mutational update of the CYP1B1 gene in PCG and reported classified mutations exon-wise, country-wise, population-wise and with respect to many other parameters. ${ }^{18}$ As is the generally accepted notion, disease causing mutations in CYP1B1 gene actually cause derangements in the protein structure in the mutant forms ensuing functional insufficiency. This means that functional characteristics of wild-type protein and its mutant forms are different; this causes alterations in enzymatic activity (in the mutants) with respect to various substrates ${ }^{34}$ leading to physiological anomalies and precipitating disease phenotype.

\section{FROM MUTATION TO LOSS OF FUNCTION}

Mutations at important positions are accompanied with the loss of function in the protein product. This is 
the conceptual basis of the mutational studies that are being carried out for various diseases. Most important mutations occur in the areas that correspond to the FIRs, like substrate binding sites, substrate access channels, heme binding regions, etc. A simple speculation for the probable mechanism is that a mutation leads to changes in the protein structure due to substitution, addition or removal of one or more amino acids and the ensuing change in charge distribution and other significant properties. Once one type of amino acid is substituted by the other (or other alterations arise), many changes occur viz surface charge distribution, protein stability, protein flexibility, folding patterns, cell trafficking, core region packing, hydrophobic interactions, catalytic site shape, substrate binding region, substrate access channel, metal binding efficiency, etc. So, an amino acid change brings in many alterations and the nature of change depends on the nature and position of the amino acid altered. Some mutations, for this reason, are well tolerated while others are not. ${ }^{35-37}$

Some investigators have devised a few important points to consider for quality assessment of the extent of deleterious nature of a mutation. ${ }^{38,39}$ The genotypephenotype correlation studies reported by Panicker et $\mathrm{al}^{40}$ adjudge some mutations (they found in their population cohort) deleterious for protein function. This led them to report quantitative measure of severe phenotypes in the Indian population with regards to these mutations [P193L (62.5\%), E229K (80\%) and R368H (72\%)]. An important phenomenon that warrants attention is that most of PCG cases with CYP1B1 mutations present with incomplete penetrance making the understanding of the disease mechanism and mode of inheritance intricately obscure. ${ }^{25}$ This observation bolsters the endeavors for functional characterization of CYP1B1 specific mutations and the characterization of FIRs. An important application of such studies is that they will aid in revealing the etiopathomechanism of PCG and will help in identifying the potential molecular targets for therapy and preventive management.

\section{CONCLUSION}

Mutations in CYP1B1 gene are the major cause of PCG. Many mutations in CYP1B1 gene have been reported in PCG patients. A proper understanding of how these mutations lead to disease phenotype is necessary. This may help in understanding the disease and devising necessary steps to curb it. Functional characterization and molecular modeling studies provide a good hope for understanding the CYP1B1-mediated pathogenic mechanism of PCG. The whole premise works on the considerations that CYP1B1 is important in ocular development, its mutations lead to malfunction which cause maldevelopment of ocular structures leading to PCG. Mutations affect protein structure and function. Expressing the gene and its relevant mutants in heterologous hosts and subjecting to various tests is likely to yield important information that may prove essential in development of novel treatments for PCG.

\section{REFERENCES}

1. Bofinger DP, Feng L, Chi LH, Love J, Stephen FD, Sutter TR, Osteen K, Costich GTG, Batt RE, Koury ST, et al. Effect of TCDD exposure on CYP1A1 and CYP1B1 expression in explant cultures of human endometrium. Toxicol Sci 2001 Aug;62(2):299-314.

2. Hakkola J, Pasanen M, Pelkonen O, Hukkanen J, Evisalmi S, Anttila S, Rane A, Mäntylä M, Purkunen R, Saarikoski S, et al. Expression of CYP1B1 in human adult and fetal tissues and differential inducibility of CYP1B1 and CYP1A1 by Ah receptor ligands in human placenta and cultured cells. Carcinogenesis 1997 Feb;18(2):391-397.

3. Stoilov I, Akarsu AN, Sarfarazi M. Identification of three different truncating mutations in cytochrome P4501B1 (CYP1B1) as the principal cause of primary congenital glaucoma (Buphthalmos) in families linked to the GLC3A locus on chromosome 2p21. Hum Mol Genet 1997;6(4):641-647.

4. Bejjani BA, Lewis RA, Tomey KF, Anderson KL, Dueker DK, Jabak M, Astle WF, Otterud B, Leppert M, Lupski JR. Mutations in CYP1B1, the gene for cytochrome P4501B1, are the predominant cause of primary congenital glaucoma in Saudia Arabia. Am J Hum Genet 1998 Feb;62(2):325-333.

5. Buters JT, Doehmer J, Gonzalez FJ. Cytochrome P450-null mice. Drug Metab Rev 1999 May;31(2):437-447.

6. Vasiliou VI, Gonzalez FJ. Role of CYP1B1 in glaucoma. Annu Rev Pharmacol Toxicol 2008;48(1):333-358.

7. Bailey LR, Roodi N, Dupont WD, Parl FF. Association of cytochrome P450 1B1 (CYP1B1) polymorphism with steroid hormone receptor status in breast cancer. Cancer Res 1998 Nov; 58(22):5038-5041.

8. Li DN, Seidel A, Pritchard MP, Wolf CR, Friedberg T. Polymor phisms in P450 CYP1B1 affect the conversion of estradiol to the potentially carcinogenic metabolite 4-hydroxyestradiol. Pharmacogenetics 2000 Jun;10(4):343-353.

9. deLuise VP, Anderson DR. Primary infantile glaucoma (congenital glaucoma). Surv Ophthalmol 1983 Jul-Aug;28(1):1-19.

10. Gencik A, Gencikova A, Ferak V. Population genetical aspects of primary congenital glaucoma. I. Incidence, prevalence, gene frequency, and age of onset. Hum Genet 1982;61(3): 193-197.

11. Sarfarazi M, Akarsu AN, Hossain A, Turacli ME, Aktan SG, Barsoum-Homsy M, Chevrette L, Sayli BS. Assignment of a locus (GLC3A) for primary congenital glaucoma (Buphthalmos) to 2p21 and evidence for genetic heterogeneity. Genomics 1995 Nov;30(2):171-177.

12. Akarsu AN, Turacli ME, Aktan SG, Barsoum-Homsy ML, Chevrette B, Sayli S, Sarfarazi M. A second locus (GLC3B) for primary congenital glaucoma (Buphthalmos) maps to the 1p36 region. Hum Mol Genet 1996 Aug;5(8):1199-1203.

13. Stoilov IR, Sarfarazi M. The third genetic locus (GLC3C) for primary congenital glaucoma (PCG) maps to chromosome 14q24.3. Invest Ophthalmol Vis Sci 2002 Dec;43(13):3015. 
14. Stoilov I. Cytochrome P450s: coupling development and environment. Trends Genet 2001 Nov;17(11):629-632.

15. Stoilov I, Jansson I, Sarfarazi M, Schenkman JB. Roles of cytochrome p450 in development. Drug Metabol Drug Interact 2001;18(1):33-55.

16. Doshi M, Marcus C, Bejjani BA, Edward DP. Immunolocalization of CYP1B1 in normal, human, fetal and adult eyes. Exp Eye Res 2006 Jan;82(1):24-32.

17. Li N, Zhou Y, Du L, Wei M, Chen X. Overview of Cytochrome $\mathrm{P} 450$ 1B1 gene mutations in patients with primary congenital glaucoma. Exp Eye Res 2011 Nov;93(5):572-579.

18. Faiq MA, Sharma R, Dada R, Mohanty K, Saluja D, Dada T. Genetic, biochemical and clinical insights into primary congenital glaucoma. J Curr Glaucoma Prac 2013 MayAug;7(2):66-84.

19. Plasilova M, Gerinec A, Ferak V, Molecular diagnosis of mutations responsible for recurrent and severe forms of primary congenital glaucoma. Cesk Slov Oftalmol 1998;54(5): 281-288.

20. Plasilova M, Ferakova E, Kadasi L, Polakova H, Gerinec A, Ott J, Ferak V. Linkage of autosomal recessive primary congenital glaucoma to the GLC3A locus in Roms (Gypsies) from Slovakia. Hum Hered 1998 Jan-Feb;48(1):30-33.

21. Stoilov I, Akarsu AN, Alozie I, Child A, Barsoum-Homsy M, Tyracli MA, Or M, Lewis RA, Ozdemir N, Brice GA, et al. Sequence analysis and homology modeling suggest that primary congenital glaucoma on 2p21 results from mutations disrupting either the hinge region or the conserved core structures of cytochrome P4501B1. Am J Hum Genet 1998 Mar;62(3):573-584.

22. Kakiuchi T, Isashiki Y, Nakao K, Sonoda S, Kimura K, Ohba N. A novel truncating mutation of cytochrome P4501B1 (CYP1B1) gene in primary infantile glaucoma. Am J Ophthalmol 1999 Sep;128(3):370-372.

23. Bejjani BA, Stockton DW, Lewis RA, Tomey KF, Dueker DK, Jabak M, Astle WF, Lupski JR. Multiple CYP1B1 mutations and incomplete penetrance in an inbred population segregating primary congenital glaucoma suggest frequent de novo events and a dominant modifier locus. Hum Mol Genet 2000 Feb;9(3):367-374.

24. Martin SN, Sutherland J, Levin AV, Klose R, Priston M, Heon E. Molecular characterisation of congenital glaucoma in a consanguineous Canadian community: a step towards preventing glaucoma related blindness. J Med Genet 2000 Jun;37(6):422-427.

25. Ohtake Y, Kubota R, Tanino T, Miyata H, Mashima Y. Novel compound heterozygous mutations in the cytochrome P4501B1 gene (CYP1B1) in a Japanese patient with primary congenital glaucoma. Ophthalmic Genet 2000 Sep;21(3): 191-193.

26. Mashima Y, Suzuki Y, Sergeev Y, Ohtake Y, Tanino T, Kimura I, Miyata H, Aihara M, Tanihara H, Inatani M, et al. Novel cytochrome P4501B1 (CYP1B1) gene mutations in
Japanese patients with primary congenital glaucoma. Invest Ophthalmol Vis Sci 2001 Sep;42(10):2211-2216.

27. Stoilov IR, Costa VP, Vasconcellos JP, Melo MB, Betinjane AJ, Carani JC, Oltrogge EV, Sarfarazi M. Molecular genetics of primary congenital glaucoma in Brazil. Invest Ophthalmol Vis Sci 2002 Jun;43(6):1820-1827.

28. Panicker SG, Reddy AB, Mandal AK, Ahmed N, Nagarajaram HA, Hasnain SE, Balasubramanian D. Identification of novel mutations causing familial primary congenital glaucoma in Indian pedigrees. Invest Ophthalmol Vis Sci 2002 May;43(5):1358-1366.

29. Reddy AB, Kaur K, Mandal AK, Panicker SG, Thomas R, Hasnain SE, Balasubramanian D, Chakrabarti S. Mutation spectrum of the CYP1B1 gene in Indian primary congenital glaucoma patients. Mol Vis 2004 Sep;10:696-702.

30. Tanwar M, Dada T, Sihota R, Dada R. Identification of four novel cytochrome P4501B1 mutations (p.I94X, p.H279D, p.Q340H, and p.K433K) in primary congenital glaucoma patients. Mol Vis 2009 Dec;15:2926-2937.

31. Tanwar M, Dada T, Sihota R, Das TK, Yadav U, Dada R. Mutation spectrum of CYP1B1 in north Indian congenital glaucoma patients. Mol Vis 2009 Jun;15:1200-1209.

32. Faiq MA, Dada R, Sharma R, Saluja D, Dada T. CYP1B1: A unique gene with unique characteristics. Curr Drug Metab 2014;15(9):893-914.

33. Faiq MA, Ali M, Dada T, Dada R, Saluja D. A novel methodology for enhanced and consistent heterologous expression of unmodified human cytochrome P450 1B1 (CYP1B1). PLoS One 2014 Oct 16;9(10):e110473.

34. Choudhary D, Jansson I, Sarfarazi M, Schenkman JB. Characterization of the biochemical and structural phenotypes of four CYP1B1 mutations observed in individuals with primary congenital glaucoma. Pharmacogenet Genomics 2008 Aug;18(8):665-676.

35. Nagarajaram HA, Reddy BV, Blundell TL. Analysis and prediction of inter-strand packing distances between betasheets of globular proteins. Protein Eng 1999 Dec;12(12): 1055-1062.

36. Reddy BV, Blundell TL. Packing of secondary structural elements in proteins. Analysis and prediction of inter-helix distances. J Mol Biol 1993 Oct;233(3):464-479.

37. Reddy BV, Nagarajaram HA, Blundell TL. Analysis of interactive packing of secondary structural elements in alpha/beta units in proteins. Protein Sci 1999 Mar;8(3): 573-586.

38. Sunyaev S, Ramensky V, Koch I, Lathe W, Kondrashov AS, Bork P. Prediction of deleterious human alleles. Hum Mol Genet 2001 Mar;10(6):591-597.

39. Wang Z, Moult J. SNPs, protein structure, and disease. Hum Mutat 2001 Apr;17(4):263-270.

40. Panicker SG, Mandal AK, Reddy AB, Gothwal VK, Hasnain SE. Correlation of genotype with phenotype in Indian patients with primary congenital glaucoma. Invest Ophthalmol Vis Sci 2004 Apr;45(4):1149-1156. 